\title{
MIGRATION OF INTERPLANETARY DUST
}

\author{
S. I. Ipatov ${ }^{1}$, J. C. Mather ${ }^{2}$, P. A. Taylor ${ }^{3}$ \\ ${ }^{1}$ (1) George Mason University, USA; (2) NASA/GSFC, Mail Code 685, Greenbelt, MD 20771, \\ USA; E-mail: siipatov@hotmail.com (for correspondence); (3) Institute of Applied \\ Mathematics, Moscow, Russia \\ ${ }^{2}$ NASA/GSFC, Mail Code 685, Greenbelt, MD 207r7, USA \\ ${ }^{3}$ University of Maryland, College Park, MD 20742, USA
}

\begin{abstract}
We numerically investigate the migration of dust particles with initial orbits close to those of the numbered asteroids, observed trans-Neptunian objects, and Comet Encke. The fraction of silicate asteroidal particles that collided with the Earth during their lifetime varied from $1.1 \%$ for 100 micron particles to $0.008 \%$ for 1 micron particles. Almost all asteroidal particles with diameter $d \geq 4$ microns collided with the Sun. The peaks in the migrating asteroidal dust particles' semi-major axis distribution at the $\mathrm{n}:(\mathrm{n}+1)$ resonances with Earth and Venus and the gaps associated with the 1:1 resonances with these planets are more pronounced for larger particles. The probability of collisions of cometary particles with the Earth is smaller than for asteroidal particles, and this difference is greater for larger particles.
\end{abstract}

\section{INTRODUCTION}

The orbital evolution of interplanetary dust particles originating from the trans-Neptunian belt [1]-[5], Halley- and Encke-type comets [6],[7], and asteroid families and short-period comets [8]-[10] has been numerically simulated by several authors in order to understand the distribution of dust in the solar system. Observationally, the infrared satellites IRAS and COBE detected thermal emission from dust outside the Earth's orbit [11]-[13] while Pioneers 10 and 11 measured the interplanetary dust population directly using impact detectors [14]. The main contribution to the zodiacal light is from particles with diameters of about 20 to $200 \mu \mathrm{m}$. It has been estimated that 20,000 to 40,000 tons of micrometer- to millimeter-sized dust particles fall to the Earth every year with the mass distribution of dust particles peaking at about $200 \mu \mathrm{m}$ in diameter [15]. Liou et al. [7] showed that the observed shape of the zodiacal cloud can be accounted for by a combination of about $1 / 4$ to $1 / 3$ asteroidal dust and about $3 / 4$ to $2 / 3$ cometary dust. The hypothesis that the 'kuiperoidal' dust (i.e., the dust formed in the Edgeworth-Kuiper belt) is dominant in the outer solar system explains the Pioneer and Voyager data fairly well, while fitting a sample of the COBE/DIRBE data indicates that the kuiperoidal dust contributes as much as $1 / 3$ of the total number density near the Earth (for a review, see [4]). Ozernoy [4] considered $1 \mathrm{~m}$ and $5 \mathrm{~m}$ particles while constructing the brightness of a disk of asteroidal, cometary, and kuiperoidal dust particles which fit the COBE/DIRBE data. 
Analysis of the Pioneer 10 and 11 meteoroid detector data [16], [17] showed that a population of $10^{-9}$ and $10^{-8} \mathrm{~g}(\sim 10 \mu \mathrm{m})$ particles has a constant spatial density between 3 and 18 AU. Dust grains released by Halley-type comets cannot account for this observed distribution [6], but trans-Neptunian dust particles can [2]. Using trans-Neptunian dust particles, Moro-Martin and Malhotra [5, Fig. 13] found that the surface (not spatial) density was approximately constant in this region.

Liou et al. [1] noted that interstellar dust particles with an average size of $1.2 \mu \mathrm{m}$ can destroy dust particles formed in the solar system and that the collisional lifetimes for 1, 2, 4, 9, $23 \mu \mathrm{m}$ particles are 104, 49, 19, 4.8, 0.86 Myr, respectively. In these size ranges mutual collisions are not as important as collisions with interstellar grains. Moro-Martin and Malhotra [5] concluded that collisional destruction is most important for kuiperoidal grains between $6 \mu \mathrm{m}(9 \mu \mathrm{m}$ in Liou et al.) and $50 \mu \mathrm{m}$. Particles larger than $50 \mu \mathrm{m}$ may survive because interstellar grains are too small to destroy them in a single impact. For silicate particles $1-40 \mu \mathrm{m}$ in diameter, the sublimation temperature $(\sim 1500 \mathrm{~K})$ is reached at $R<0.5 \mathrm{AU}$, but for water ice particles the sublimation temperature $(\sim 100 \mathrm{~K})$ is reached at $27,19,14,10$, and $4.3 \mathrm{AU}$ for the sizes of 3,6 , 11,23 , and $120 \mu \mathrm{m}$, respectively [1],[5].

In the present paper we consider a different set of initial orbits for the dust particles than the aforementioned authors and, for the first time, investigate the collisional probabilities of migrating particles with the planets based on a set of orbital elements during their evolution. We also present plots of the orbital elements of the migrating particles.

\section{MODELS FOR ASTEROIDAL DUST PARTICLES}

Using the Bulirsh-Stoer method of integration, we investigated the migration of asteroidal dust particles under the influence of planetary gravity (excluding Pluto), radiation pressure, Poynting-Robertson drag, and solar wind drag for values of the ratio between the radiation pressure force and the gravitational force $\beta$ equal to $0.004,0.01,0.05,0.1,0.25$, and 0.4. Burns et al. [18] obtained $\beta=0.573 Q_{p r} /(\rho s)$, where $\rho$ is the particle's density in grams per cubic centimeter, $s$ is its radius in micrometers, and $Q_{p r}$ is the radiation pressure coefficient $\left(Q_{p r}\right.$ is close to unity for particles larger than $1 \mu \mathrm{m})$. For silicates, the $\beta$ values $0.004,0.01,0.05$, $0.1,0.25$, and 0.4 correspond to particle diameters of about 100,40,9, 4, 1.6, and 1 microns, respectively. Silicate particles with $\beta$ values of 0.01 and 0.05 have masses of $10^{-7} \mathrm{~g}$ and $10^{-9}$ g. For water ice, our $\beta$ values correspond to particle diameters of $300,120,23,11,6$, and $3 \mu \mathrm{m}$ [18]. We assume the ratio of solar wind drag to Poynting-Robertson drag to be 0.35 [5], [6]. The relative error per integration step was taken to be less than $10^{-8}$.

The initial positions and velocities of the asteroidal particles were the same as those of the first numbered main-belt asteroids (JDT 2452500.5), i.e., dust particles are assumed to leave the asteroids with zero relative velocity. We considered $N=500$ particles for each $\beta \geq 0.05, N=250$ for $\beta=0.01$, and $N=100$ for $\beta=0.004$. With $\beta \geq 0.01$ in each run we took $N=250$, because for $N \geq 500$ the computer time per calculation for one particle was several times greater than for $N=250$. The simulations continued until all of the particles either collided with the Sun or reached $2000 \mathrm{AU}$ from the Sun. The lifetimes of all considered asteroidal dust particles were less than 0.8 Myr, except for one particle with a lifetime of 19 Myr. We also made similar runs without planets to investigate the role of planets in interplanetary dust migration.

\section{COLLISIONS of ASTEROIDAL PARTICLES WITH PLANETS AND THE SUN}

In our runs, planets were considered as material points, but using orbital elements obtained with a step $d_{t}$ of $\leq 20 \mathrm{yr}\left(d_{t}=10 \mathrm{yr}\right.$ for $\beta$ equal to 0.1 and 0.25 , and $d_{t}=20 \mathrm{yr}$ for other values of $\beta$ ), similar to [19] we calculated the mean probability $P=P_{\Sigma} / N\left(P_{\Sigma}\right.$ is the probability for all $N$ 
Table 1. Values of $T, T_{J}, T_{S}^{\min }, T_{S}^{\max }, T_{2000}^{\min }, T_{2000}^{\max }$ (in Kyr), $P_{r}$, and $P_{S u n}$ obtained for asteroidal dust particles for several values of $\beta$ (Venus $=\mathrm{V}$, Earth $=\mathrm{E}$, Mars $=\mathrm{M}$ )

\begin{tabular}{lcccccccccccc}
\hline & & $\mathrm{V}$ & $\mathrm{V}$ & $\mathrm{E}$ & $\mathrm{E}$ & $\mathrm{M}$ & $\mathrm{M}$ & & & & & \\
$\beta$ & $P_{\text {Sun }}$ & $P_{r}$ & $T$ & $P_{r}$ & $T$ & $P_{r}$ & $T$ & $T_{J}$ & $T_{S}^{\min }$ & $T_{S}^{\max }$ & $T_{2000}^{\min }$ & $T_{2000}^{\max }$ \\
\hline 0.004 & 1.000 & 12783 & 40.5 & 11350 & 90. & 1204 & 220. & 0 & 348 & 932 & & \\
0.01 & 1.000 & 1534 & 19.2 & 1746 & 44.2 & 127 & 99.9 & 0 & 142 & 422 & & \\
0.05 & 0.996 & 195 & 4.0 & 190 & 8.1 & 36.7 & 20.5 & 0 & 30 & 89 & & \\
0.1 & 0.990 & 141 & 2.4 & 132 & 4.8 & 16.4 & 12.0 & 2.21 & 16 & 44 & 138 & 793 \\
$0.1 *$ & 0.990 & 366 & 2.4 & 279 & 4.8 & 20.9 & 12.0 & 0.92 & 7.2 & 43 & 9 & 534 \\
0.25 & 0.618 & 79.2 & 1.4 & 63.8 & 2.9 & 5.60 & 5.9 & 31.7 & 5.9 & 385 & 1.6 & 567 \\
0.4 & 0.316 & 12.4 & 1.5 & 8.0 & 2.5 & 0.72 & 8.8 & 32.3 & 4.3 & 172 & 1.7 & 288 \\
\hline
\end{tabular}

considered particles) of a collision of a particle with a planet during the lifetime of the particle. We define $T=T_{\Sigma} / N$ as the mean time during which the perihelion distance $q$ of a particle was less than the semi-major axis of the planet and $T_{J}$ as the mean time spent in Jupiter-crossing orbits. Below, $P_{\text {Sun }}$ is the ratio of the number of particles that collided with the Sun to the total number of particles. $T_{S}^{\min }$ and $T_{S}^{\max }$ are the minimum and maximum values of the time until the collision of a particle with the Sun, and $T_{2000}^{\min }$ and $T_{2000}^{\max }$ are the minimum and maximum values of the time when the distance between a particle and the Sun reached 2000 AU. With $\beta \geq 0.01$ the values of $P_{r}=10^{6} P, T, T_{J}, T_{S}^{\min }, T_{S}^{\max }, T_{2000}^{\min }$, and $T_{2000}^{\max }$ are shown in Table 1 for $N=250$ (for $\beta=0.1$ we present two runs with 250 different particles), and $P_{S u n}$ was obtained for all considered particles at a fixed $\beta$.

The minimum time $T_{S}^{\min }$ needed to reach the Sun is smaller for smaller particles, but the ratio $T_{S}^{\max } / T_{S}^{\min }$ is much greater and the ratio $T_{2000}^{\max } / T_{S}^{\max }$ is smaller for $\beta \geq 0.25$ than for $\beta \leq 0.1$. For $\beta=0.05,498$ particles collided with the Sun in less than $0.089 \mathrm{Myr}$, but two particles (with initial orbits close to those of the asteroids 361 and 499), which reached $2000 \mathrm{AU}$, lived for $0.21 \mathrm{Myr}$ and 19.06 Myr, respectively. The latter object's perihelion was near Saturn's orbit for a long time. At $\beta=0.05$ the first 250 particles did not migrate outside Jupiter's orbit, so $T_{J}=0$ in Table 1.

For smaller particles (i.e., those with larger $\beta$ ), $P_{\text {Sun }}$ is smaller and the probability of collisions of particles with the terrestrial planets is smaller. The probability of a collision of a migrating dust particle with the Earth for $\beta=0.004$ is greater by a factor of 1420 than for $\beta=0.4$. These probabilities of collisions are in accordance with cratering records in lunar material and on the panels of the Long Duration Exposure Facility, which showed that the mass distribution of dust particles encountering Earth peaks at $d=200 \mu \mathrm{m}[9]$.

\section{ORBITAL EVOLUTION OF ASTEROIDAL PARTICLES}

Several plots of the distribution of migrating asteroidal particles in their orbital elements and the distribution of particles with their distance $R$ from the Sun and their height $h$ above the initial plane of the Earth's orbit are presented in Figs. 1-7 (Fig. 8 present the results for kuiperoidal dust particles). For Fig. 1-2 the number of bins in the semi-major axis $a$ is 1000 . For the other figures the number of bins in $a$ or $R$ is 100, and the number of bins in $e$ or $h$ is usually slightly less than 100 . The width of a bin in $i$ is $1^{\circ}\left(0.5^{\circ}\right.$ for Fig. 8).

In Figs. 1-2, 4-8 to calculate the orbital elements we included the coefficient (1- $\beta)$ on the mass of the Sun, because of radiation pressure. Fig. 3 is the only exception. Although similar to Fig. 
4, Fig. 3 (see also figures in [20]) shows the osculating orbital elements that are calculated using the normal mass of the Sun (i.e., to transfer from rectangular coordinates to orbital elements we used the same formulas as those for massive bodies). For transformations between semimajor axes and eccentricities in the above two systems of orbital elements we used the formulas obtained by Kortenkamp and Dermott [9]. In total, Figs. 3 and 4 are similar, but they differ in some details. For example, for $\beta=0.4$ in Fig. 4 there are some pairs of $a$ and $e$ corresponding to a perihelion near Saturn's orbit. There are no such pairs in Fig. 3 for $\beta=0.4$.

We obtained that the mean time $t_{a}$ (the total time divided by the number $N$ of particles) during which an asteroidal dust particle had a semi-major axis $a$ in an interval of fixed width is greater for smaller $\beta$ at semi-major axes $a<3 \mathrm{AU}$ (exclusive of the gap at $a=1 \mathrm{AU}$ and $\beta=0.01$ ). In Fig. 1b curves plotted at $40 \mathrm{AU}$ are (top-to-bottom) for $\beta$ equal to $0.25,0.4,0.05,0.1$, and 0.01 . For $\beta \leq 0.1$ the values of $t_{a}$ are much smaller at $a>3.5 \mathrm{AU}$ than at $1<a<3 \mathrm{AU}$, and they are usually a maximum at $a \approx 2.3 \mathrm{AU}$. For $\beta=0.01$ the local maxima of $t_{a}$ corresponding to the $6: 7,5: 6,3: 4$, and 2:3 resonances with the Earth are greater than the maximum at $2.4 \mathrm{AU}$. There are several other local maxima (Fig. 1c) corresponding to the $\mathrm{n}:(\mathrm{n}+1)$ resonances with Earth and Venus (e.g., the 7:8 and 4:5 resonances with Venus). The trapping of dust particles in the $\mathrm{n}:(\mathrm{n}+1)$ resonances cause Earth's asteroidal ring [21], [22]. The greater the $\beta$, the smaller the local maxima corresponding to these resonances. At $\beta \leq 0.1$ there are gaps with $a$ a little smaller than the semi-major axes of Venus and Earth that correspond to the 1:1 resonance for each; the greater the $\beta$, the smaller the corresponding values of $a$. A small gap for Mars is seen only at $\beta=0.01$. There are also gaps corresponding to the $3: 1,5: 2$, and $2: 1$ resonances with Jupiter.

For all considered $\beta, t_{a}$ decreases considerably with a decrease of $a$ at $a<1$ AU and usually decreases with an increase of $a$ at $a>5 \mathrm{AU}$ (Fig. 1). Relatively large values of $t_{a}$ at $a>40 \mathrm{AU}$ for $\beta=0.05$ are due to one particle. For $a>5 \mathrm{AU}$ the values of $t_{a}$ are usually a little greater at $\beta=0.25$ than those at $\beta=0.4$. The number of particles with $a>5 \mathrm{AU}$ at $\beta=0.25$ is smaller than at $\beta=0.4$, but they move more slowly to $2000 \mathrm{AU}$ than at $\beta=0.4$. Analyzing Fig. 1a, we can conclude that larger particles make up a greater proportion of the dust population in the terrestrial zone than they do in the asteroid belt.

In Fig. 2 we present the results obtained for the model without planets. In this case, migration outside $5 \mathrm{AU}$ is smaller than in the model with planets and, of course, there are no peaks and gaps caused by planets (see Fig. 1). For the model without planets, the values of $T_{S}^{\min }$ were about the same as those presented in Table 1, but the values of $T_{S}^{\max }$ sometimes were smaller. With $N=250$ the values of $P_{\text {Sun }}$ for $\beta=0.25$ and $\beta=0.4$ of 0.908 and 0.548 , respectively, are greater than for the model with planets. For $\beta \leq 0.1$ all of the particles collided with the Sun.

We now return to the model with planets. At $a<4$ AU the maximum eccentricities for $\beta \geq 0.25$ were greater than those for $\beta \leq 0.1$ (Figs. 4-5). At $\beta=0.01$ some particles migrated into the 1:1 resonance with Jupiter. For $a>10$ AU perihelia were usually near Jupiter's orbit (for $\beta=0.05$ and $\beta=0.25$ also near Saturn's orbit). In almost all cases, the inclinations $i<50^{\circ}$; at $a>10 \mathrm{AU}$ the maximum $i$ was smaller for smaller $\beta$ (Fig. 6).

In Fig. 5 we present the distributions of dust particles in $a$ and $e$, and in $a$ and $i$ for $\beta=0.1$. The plots on the left were obtained for initial positions and eccentricities close to those of the asteroids with numbers 1-250, and the plots on the right, to those of the asteroids with numbers 251-500 (Figs. 1-4, 6-7 were obtained using all particles). Overall, the left and the right plots are similar, but for the left plots particles spent more time outside $5 \mathrm{AU}$. At $\beta=0.05$ none of the first 250 particles reached Jupiter's orbit, but two particles with numbers 361 and 499 migrated to 2000 AU with perihelia near the orbits of Jupiter and Saturn (Fig. 4).

Usually there are no particles with $h / R>0.7$ at $R<10 \mathrm{AU}$, with $h / R>0.25$ at $R>20$ AU for 

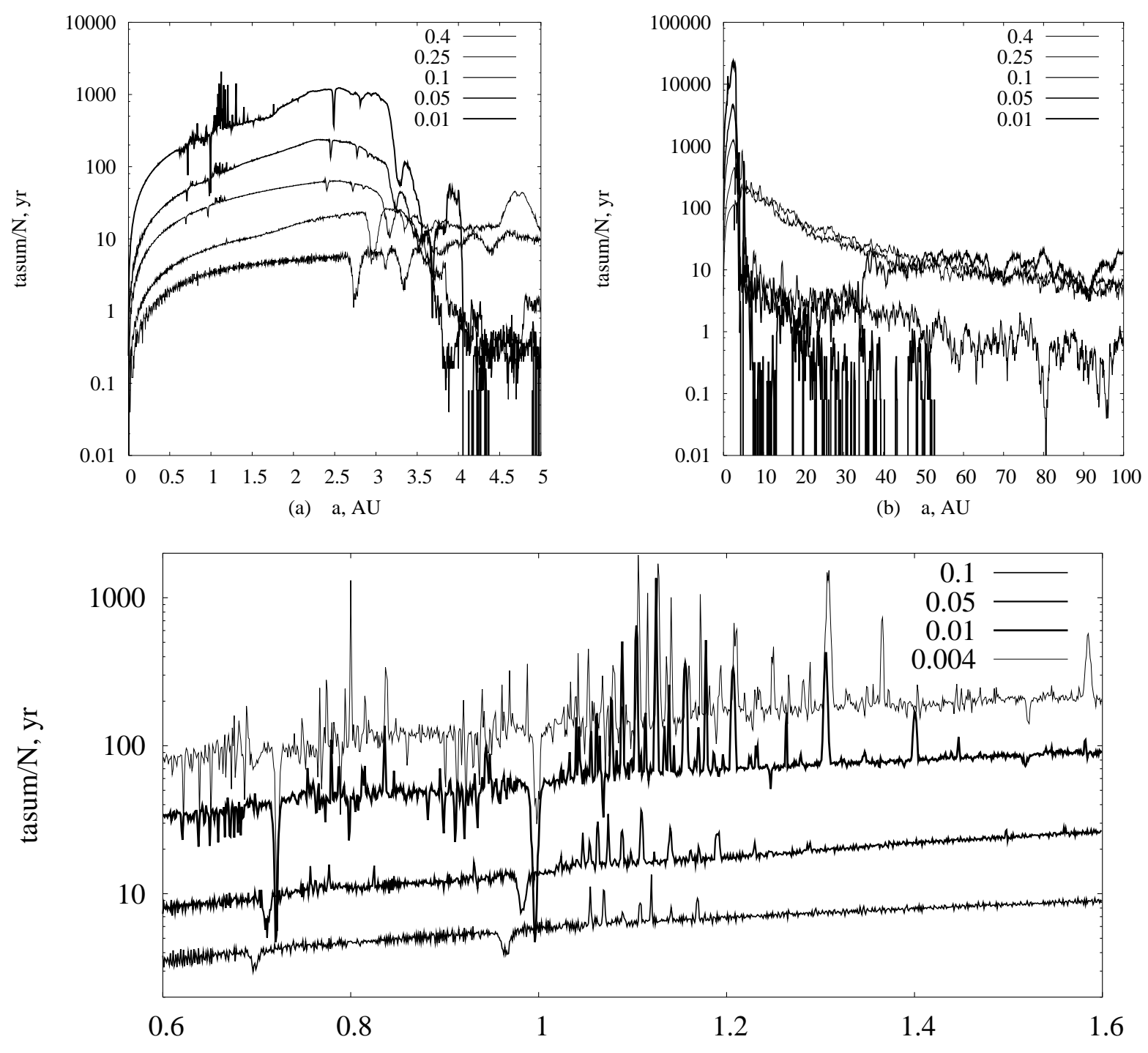

(c) a, AU

Fig. 1. Mean time $t_{a}$ (the total time tasum divided by the number $N$ of particles) during which an asteroidal dust particle had a semi-major axis in an interval with a width of (a) $0.005 \mathrm{AU}$, (b) 0.1 $\mathrm{AU}$, or (c) $0.001 \mathrm{AU}$. The values of $t_{a}$ at $1.5 \mathrm{AU}$ are greater for smaller $\beta$. Curves plotted at $40 \mathrm{AU}$ are (top-to-bottom) for $\beta$ equal to $0.25,0.4,0.05,0.1$, and 0.01 . For calculations of orbital elements we added the coefficient $(1-\beta)$ to the mass of the Sun, which is due to the radiation pressure (the same orbital elements are used in Figs. 1-2, 4-8). Initial velocities and coordinates of dust particles were the same as those of the first $N=500$ numbered asteroids $(N=250$ for $\beta=0.01, N=100$ for $\beta=0.004)$ at JDT 2452500.5 . 


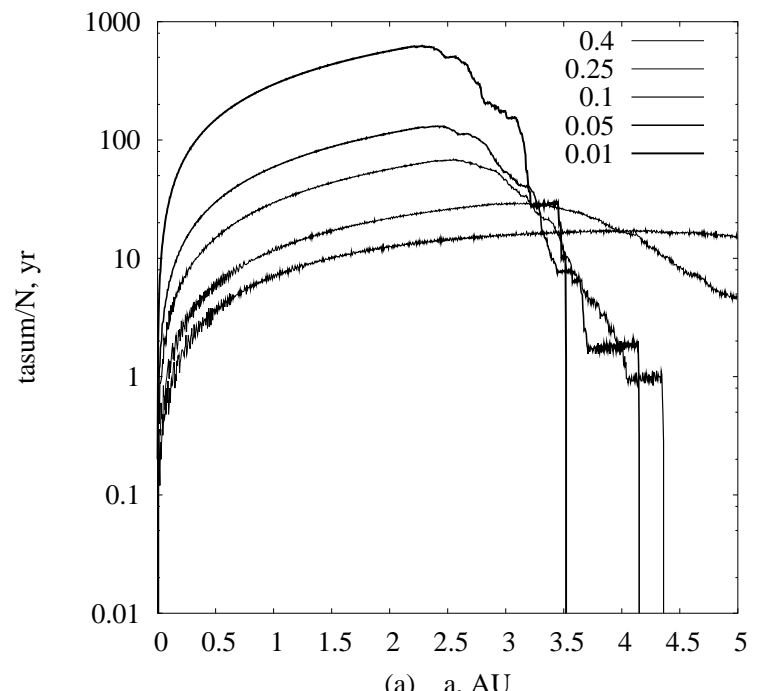

(a) a, AU

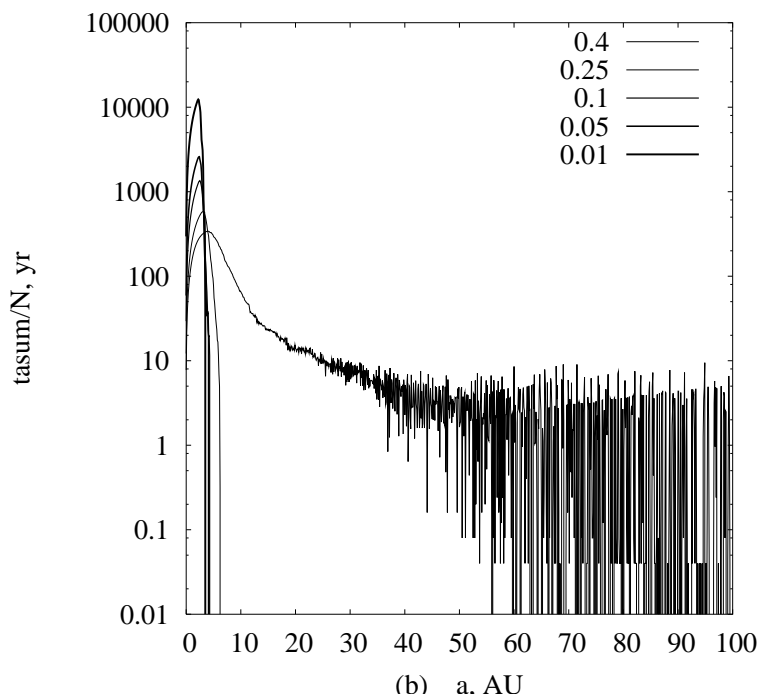

(b) $\mathrm{a}, \mathrm{AU}$

Fig. 2. Same as for Fig. 1 but without gravitational influence of planets and for a smaller number of initial dust particles $(N=250$ at $\beta \geq 0.05$, and $N=100$ at $\beta=0.01$ ). The values of $a>6.2$ AU were reached only at $\beta=0.4$.

$\beta \leq 0.1$, and with $h / R>0.5$ at $R>50$ AU for $\beta \geq 0.25$. For $\beta \geq 0.25$ at $R<1000$ AU the entire region with $h / R<0.3$ was not empty (Fig. 7 ).

The total time spent by 250 particles in inner-Earth $(Q=a(1+e)<0.983 \mathrm{AU})$, Aten $(a<1$ AU, $Q>0.983 \mathrm{AU})$, Apollo $(a>1 \mathrm{AU}, q=a(1-e)<1.017 \mathrm{AU})$, and Amor $(a>1 \mathrm{AU}$, $1.017<q<1.300 \mathrm{AU})$ orbits was 5.6, 1.4, 4.5, and $7.5 \mathrm{Myr}$ at $\beta=0.01$ and 0.09, 0.08, 0.48, and $0.76 \mathrm{Myr}$ at $\beta=0.4$, respectively. The spatial density of a dust cloud and its luminosity (as seen from outside) were greater for smaller $R$. For example, depending on $\beta$ they were by a factor of $2.5-8$ and $7-25$ ( 4 and $12-13$ at $\beta \leq 0.05$ ) greater at 1 AU than at $3 \mathrm{AU}$ for the spatial density and luminosity, respectively. This is in accordance with the observations for the inner solar system: inversion of zodiacal light observations by the Helios spaceprobe revealed a particle density $n(R) \propto R^{-1.3}$, Pioneer 10 observations between the Earth's orbit and the asteroid belt yielded $n(R) \propto R^{-1.5}$, and IRAS observations have yielded $n(R) \propto R^{-1.1}$ [23]. The intensity $I$ of zodiacal light falls off with heliocentric distance $R$ as $I \sim R^{-\gamma}$, with $\gamma=2$ to 2.5 and beyond about $3 \mathrm{AU}$ zodiacal light was no longer observable above the background light [17]. As in [2], we approximately defined the brightness of each particle as $R^{-2}$. Beyond Jupiter's orbit even the number of asteroidal particles at some distance $R$ from the Sun is smaller for greater $R$, so asteroidal dust particles cannot explain the constant spatial density of dust particles at $R \sim 3-18$ AU. At such distances, many of the dust particles could have come from the trans-Neptunian belt or from passing comets.

\section{MIGRATION OF KUIPEROIDAL DUST PARTICLES}

We also began a series of runs in which the initial positions and velocities of the particles were the same as those of the first trans-Neptunian objects (JDT 2452600.5), and our initial data were different from those in previous papers. We store orbital elements with a step of $100 \mathrm{yr}$. At the present time these runs have been finished for $\beta=0.1$ and $\beta=0.2$ with $N=50$.

The distributions of dust particles with semi-major axis $a$ and eccenticity $e$ or inclination $i$, 

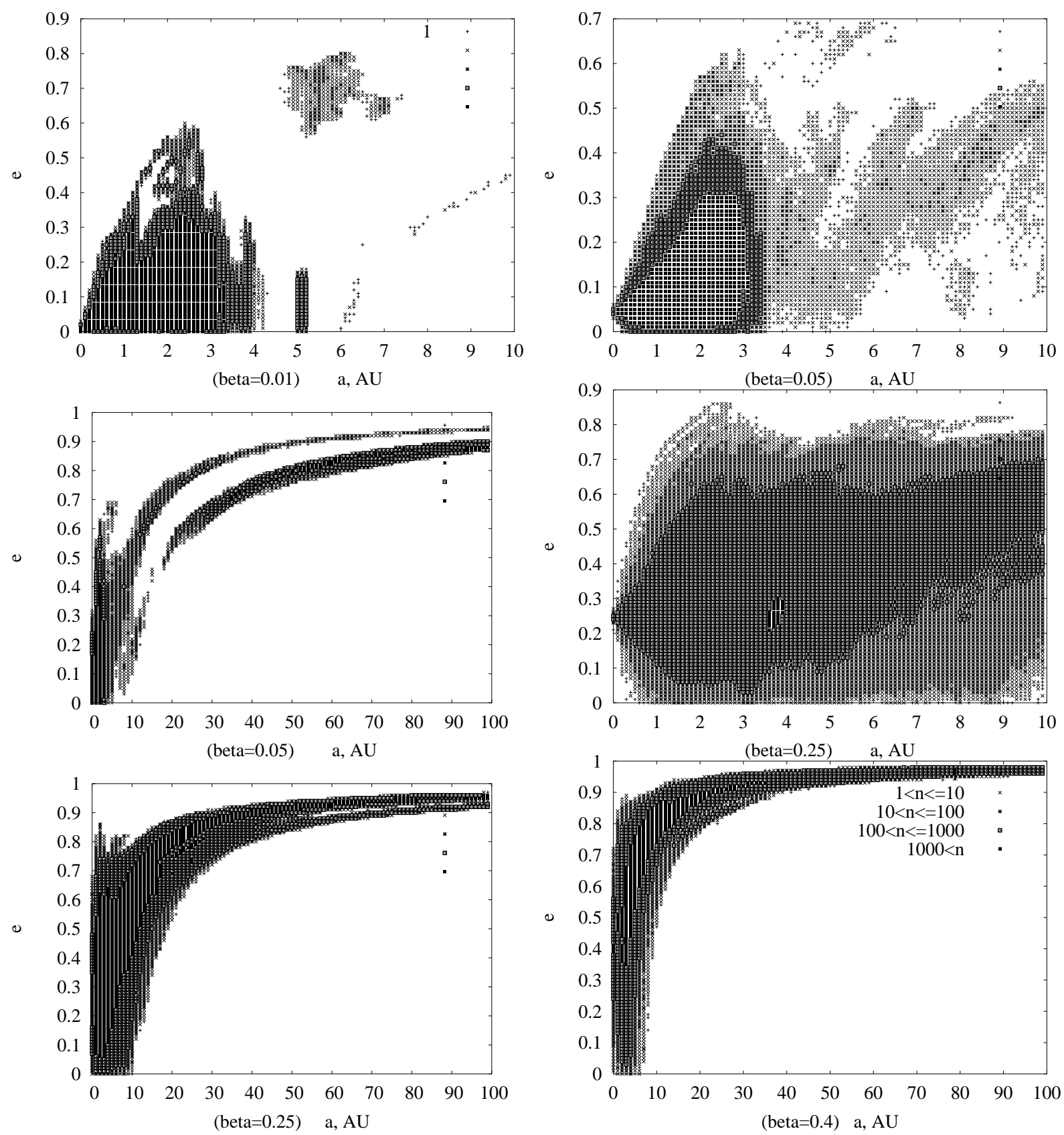

Fig. 3. Distribution of asteroidal dust particles with semi-major axis and eccentricity (designations of the number of particles in one bin are the same in Figs. 3-7). For the transfer from rectangular coordinates to orbital elements we used the same formulas as those for massive bodies. For Figs. $1-2,4-8$ for calculations of orbital elements we added the coefficient $(1-\beta)$ to the mass of the Sun, which is due to the radiation pressure. 

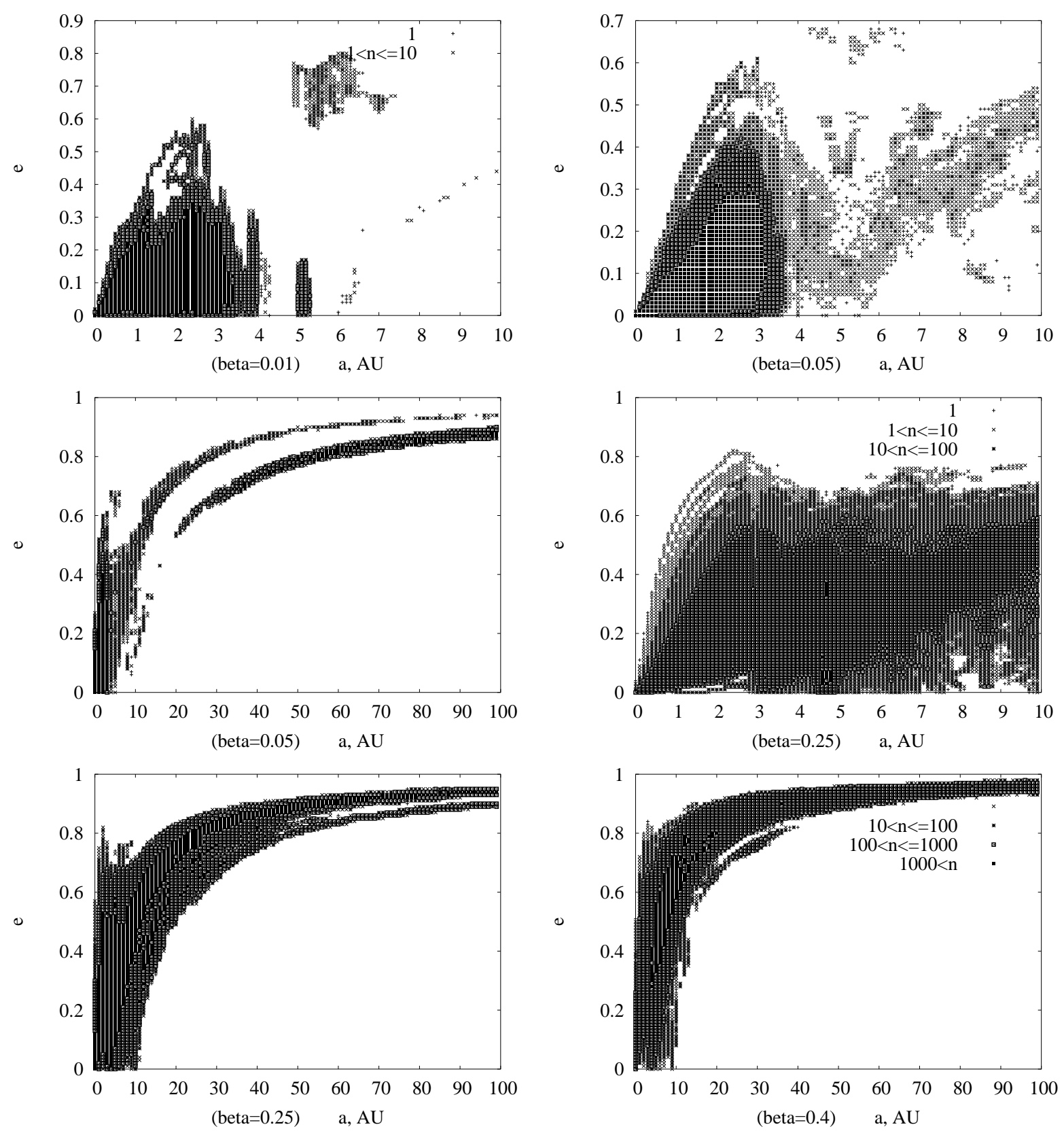

Fig. 4. Distribution of asteroidal dust particles with semi-major axis and eccentricity (designations of the number of particles in one bin are the same in Figs. 3-7). 

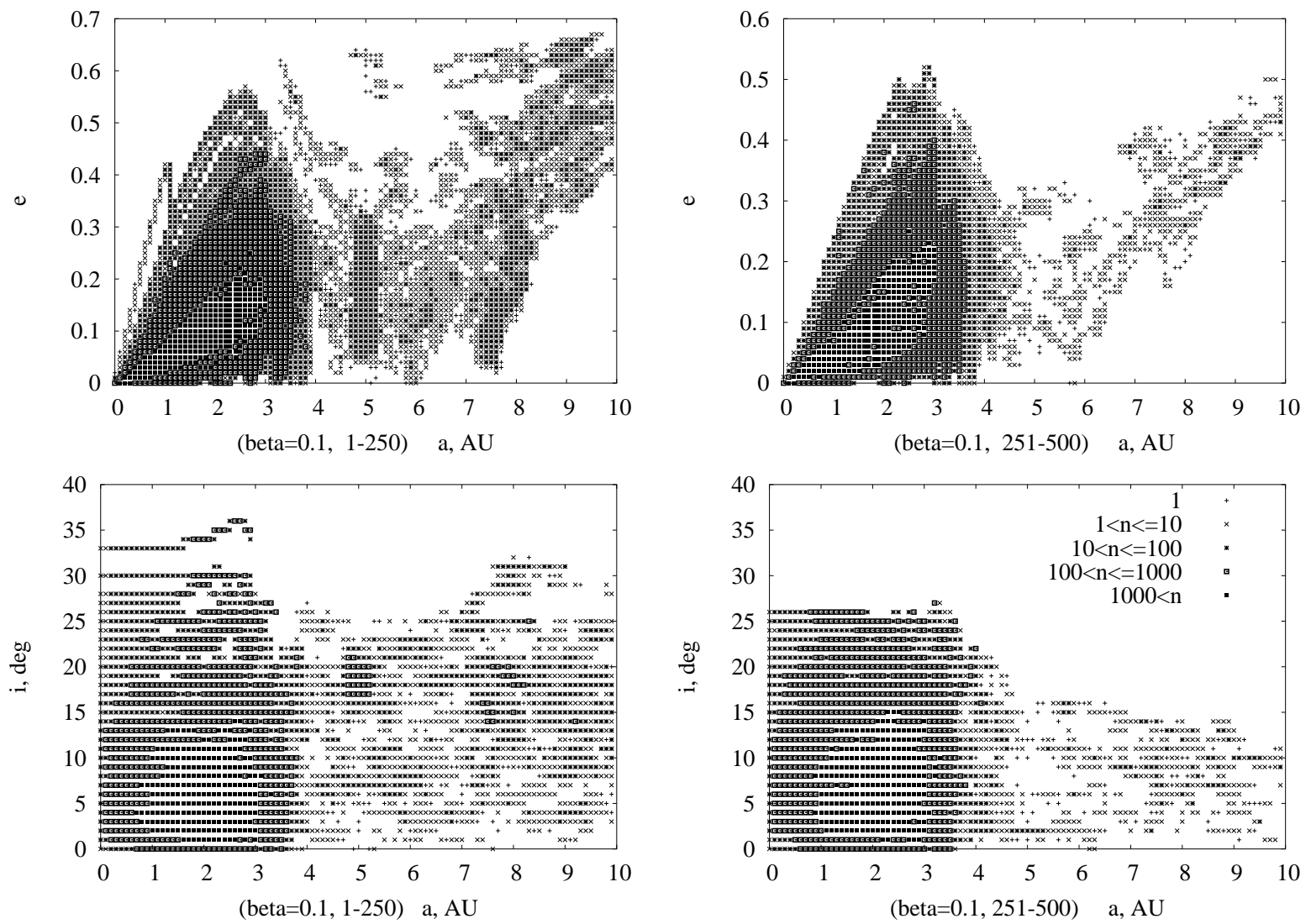

Fig. 5. Distribution of asteroidal dust particles with semi-major axis and eccentricity, and with semi-major axis and inclination for $\beta=0.1$. The left figures correspond to the runs with initial positions and velocities close to those of the first 250 numbered main-belt asteroids, and the right figures correspond to the runs with initial positions and velocities close to those of the asteroids with numbers 251-500 (JDT 2452500.5).
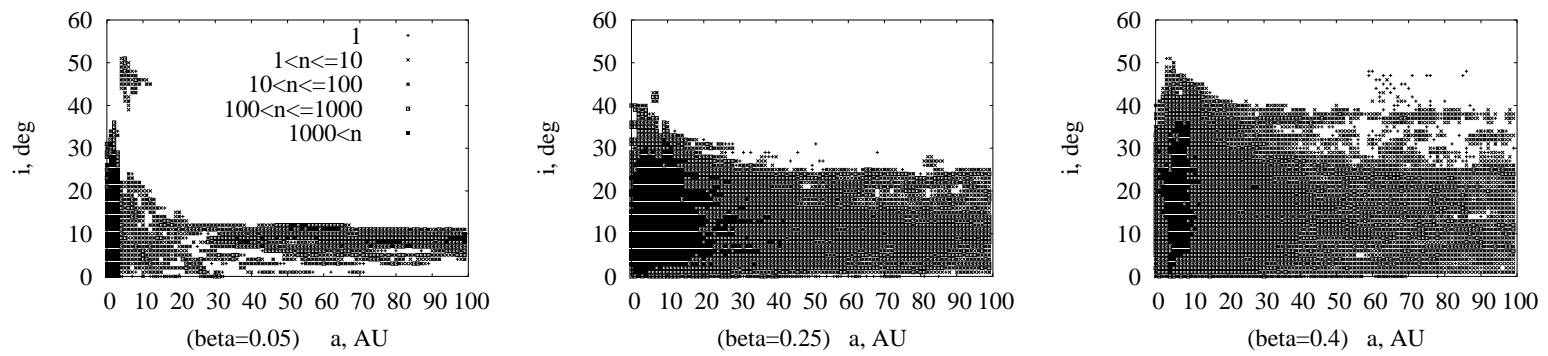

Fig. 6. Distribution of asteroidal dust particles with semi-major axis and inclination. 

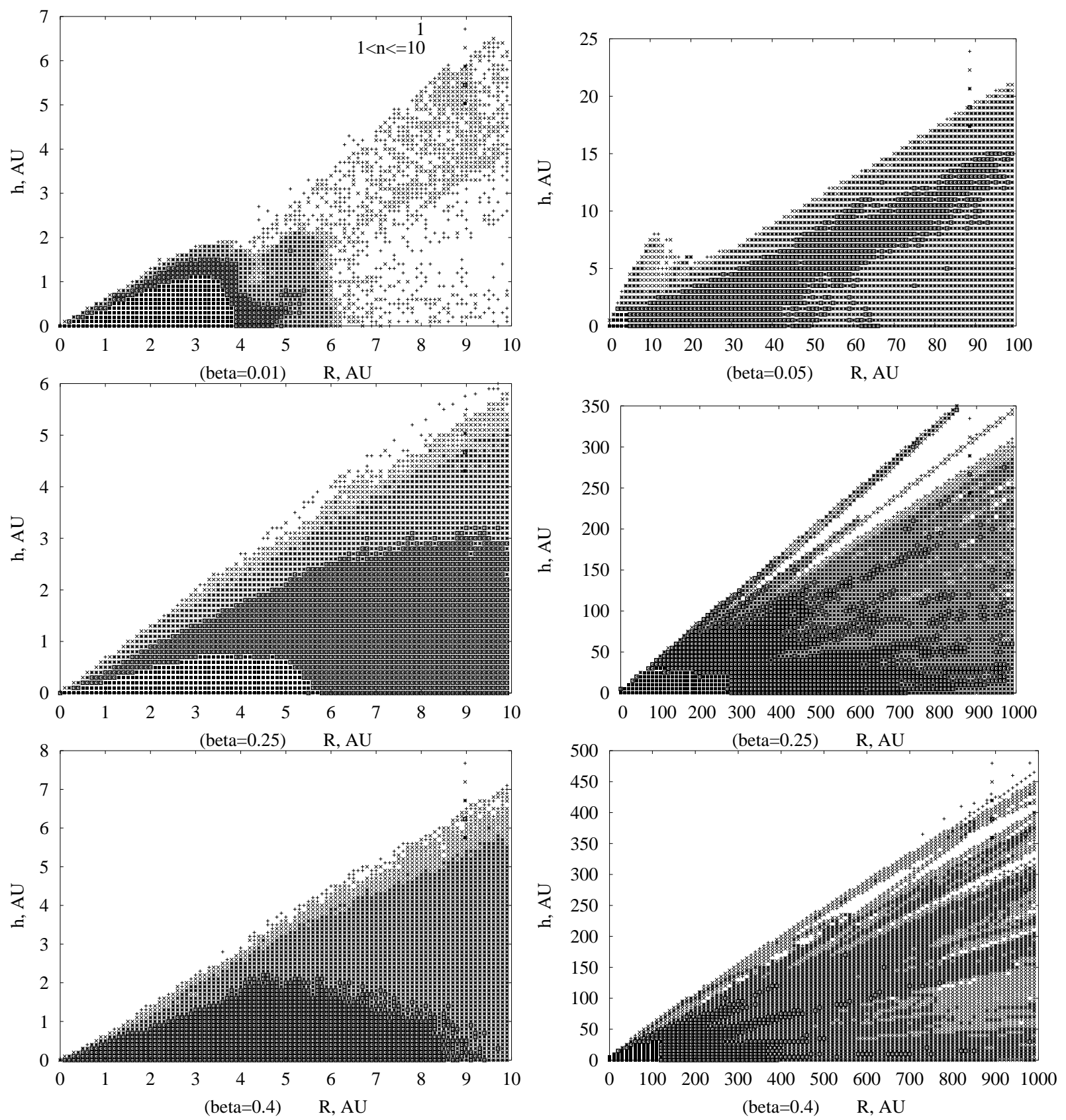

Fig. 7. Distribution of asteroidal dust particles with distance $R$ from the Sun and height $h$ above the initial plane of the Earth's orbit (designations of the number of particles in one bin are the same in Figs. 3-7). 
Table 2. Values of $T, T_{J}, T_{S}^{\min }, T_{S}^{\max }, T_{2000}^{\min }, T_{2000}^{\max }$ (in Kyr), $P_{r}$, and $P_{S u n}$ obtained for kuiperoidal dust particles at $\beta=0.1$ and $\beta=0.2$ (Venus $=\mathrm{V}$, Earth $=\mathrm{E}$, Mars $=\mathrm{M}$ )

\begin{tabular}{ccccccccccccc}
\hline & & $\mathrm{V}$ & $\mathrm{V}$ & $\mathrm{E}$ & $\mathrm{E}$ & $\mathrm{M}$ & $\mathrm{M}$ & & & & & \\
$\beta$ & $P_{\text {Sun }}$ & $P_{r}$ & $T$ & $P_{r}$ & $T$ & $P_{r}$ & $T$ & $T_{J}$ & $T_{S}^{\min }$ & $T_{S}^{\max }$ & $T_{2000}^{\min }$ & $T_{2000}^{\max }$ \\
\hline 0.1 & 0.2 & 76.2 & 0.75 & 35.2 & 1.42 & 2.74 & 2.79 & 47.2 & 3659 & 17439 & 3730 & 53949 \\
0.2 & 0.12 & 182 & 0.22 & 150 & 0.46 & 13.3 & 1.2 & 59.6 & 5237 & 10789 & 2490 & 26382 \\
\hline
\end{tabular}

and with distance $R$ from the Sun and height $h$ above the initial plane of the Earth's orbit are presented in Fig. 8. The left plots were obtained for $\beta=0.1$, and the right plots were made for $\beta=0.2$. For both values of $\beta$, particles with $e>0.5$ had their perihelia mainly near the semi-major axis of the giant planets. The mean eccentricity $e_{m}$ of kuiperoidal dust particles increased with $a$ at $a>50 \mathrm{AU}$, and it exceeded 0.5 at $a>60 \mathrm{AU}$. Bodies that migrated inside Neptune's orbit had $e_{m}<0.2$. The inclinations were usually less than $35^{\circ}$. Similar figures for intermediate stages of the runs for kuiperoidal particles were presented in [24] (for $\beta=0.05, N=50$, and a time interval of $2 \mathrm{Myr}$; and for $\beta=0.1, N=100$, and $t \leq 3.5 \mathrm{Myr}$ ).

At $\beta=0.4$ most particles were outside $50 \mathrm{AU}$ after only $0.02 \mathrm{Myr}$. If we consider positions of particles for $t \leq 0.05 \mathrm{Myr}$, then $84 \%$ of them had $R>100 \mathrm{AU}$. At that time the values of $n_{R} / R$ (surface density), $n_{R} / R^{2}$ (spatial density), and $n_{R} / R^{3}$ (similar to luminosity) were maximum at $\sim 40-55$ AU.

In Fig. 8 the values of $h$ were maximum $(30 \mathrm{AU})$ at $R \sim 57 \mathrm{AU}$ for $\beta=0.1$, and were maximum (42 AU) at $R \sim 80 \mathrm{AU}$ for $\beta=0.2$. We usually obtained $h / R<0.5$, and in the zone of the Edgeworth-Kuiper belt the ratio of the number of particles at $h=k R$ dropped usually to $10 \%$ of the number at $h=0$ at $k=0.1$, but sometimes at $k=0.25$.

Table 2 is similar to Table 1 , but was obtained for kuiperoidal dust particles. The fraction $P_{\text {Sun }}$ of kuiperoidal particles collided with the Sun was smaller by a factor of 6 than that of asteroidal particles for the same $\beta$, but the difference in collision probabilities with Earth and Venus for asteroidal and kuiperoidal particles was smaller than 6 . This is due to that the mean eccentricities of particles near these planets (especially, at $\beta=0.2$ ) were smaller for kuiperoidal particles than those for asteroidal particles.

The trans-Neptunian belt is considered to be the main source of Jupiter-family comets, which can produce much trans-Neptunian dust just inside Jupiter's orbit. Some of these comets can reach typical near-Earth objects' orbits [19]. The total mass of comets inside Jupiter's orbit is much smaller than the total mass of asteroids, but a comet produces more dust per unit minor body mass than an asteroid.

\section{MIGRATION OF DUST PARTICLES FROM COMET ENCKE}

We also investigated the migration of cometary dust particles for $\beta$ equal to $0.002,0.004$, $0.01,0.05,0.1,0.2$, and 0.4 . For silicate particles such values of $\beta$ correspond to diameters equal to about 200, 100,40,9,4,2, and 1 microns, respectively. The initial positions and velocities of the particles were the same as those of Comet $2 \mathrm{P}$ Encke. We considered particles starting near perihelion (runs denoted as $\Delta t_{o}=0$ ), near aphelion $\left(\Delta t_{o}=0.5\right.$ ), and when the comet had orbited for $P_{a} / 4$ after perihelion passage, where $P_{a}$ is the period of the comet (such runs are denoted as $\left.\Delta t_{o}=0.25\right)$. Variations in time $\tau$ when perihelion was passed was varied with a step 0.1 day for series 'S' and with a step 1 day for series 'L'. For each $\beta$ we considered $N=101$ particles for "S" runs and 150 particles for "L" runs. 

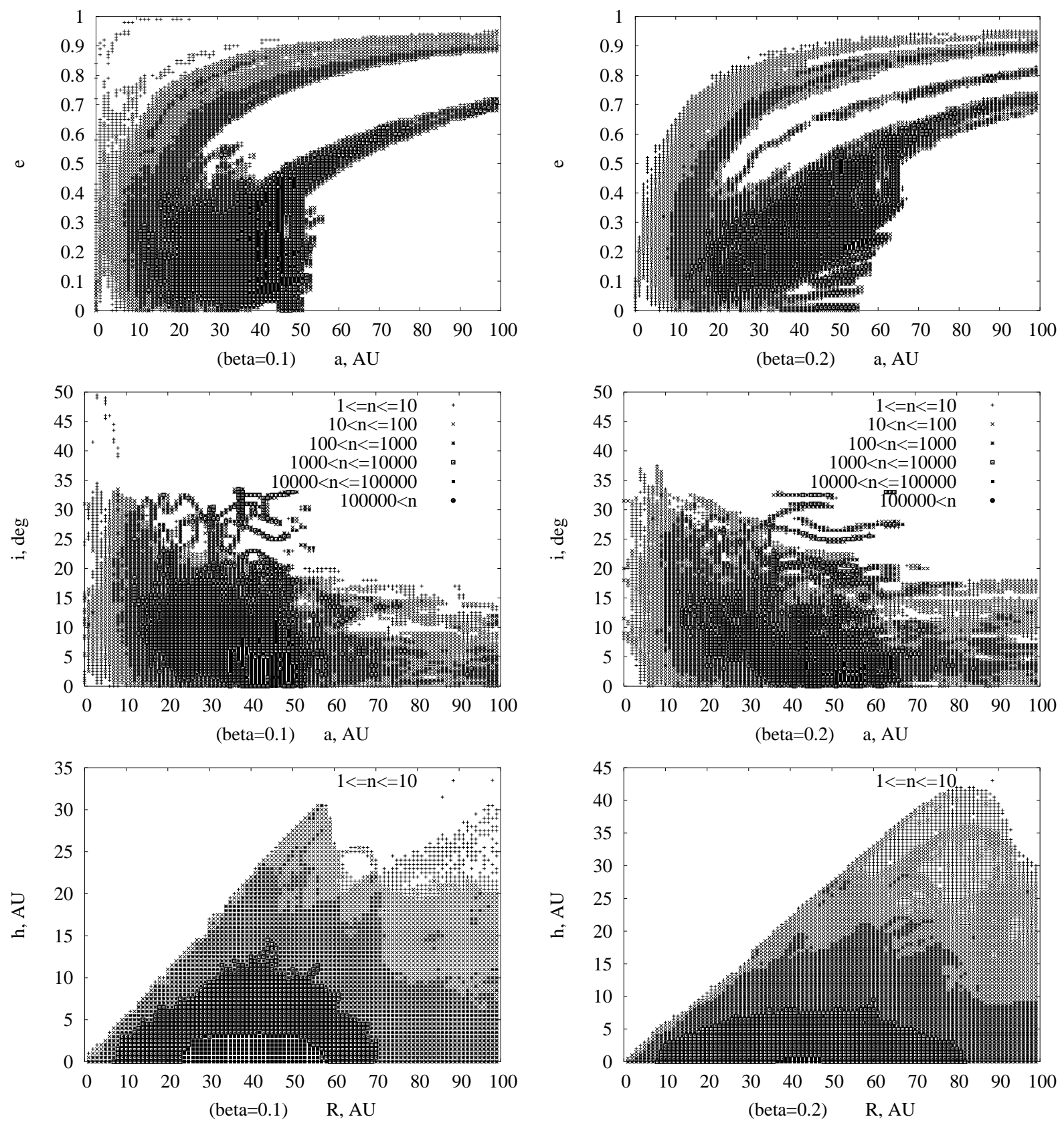

Fig. 8. Distribution of trans-Neptunian (kuiperoidal) dust particles with semi-major axis $a$ and eccenticity $e$ or inclination $i$, and with distance $R$ from the Sun and height $h$ above the initial plane of the Earth's orbit. 
Table 3. Values of $T, T_{l}$ (in Kyr), and $P_{r}$ (Venus $=\mathrm{V}$, Earth $\left.=\mathrm{E}, \mathrm{Mars}=\mathrm{M}\right)$

\begin{tabular}{lllllllc|lccccccc}
\hline & & & $\mathrm{V}$ & $\mathrm{E}$ & $\mathrm{E}$ & $\mathrm{M}$ & & \multicolumn{1}{c}{$\mathrm{V}$} & $\mathrm{E}$ & $\mathrm{E}$ & $\mathrm{M}$ \\
$\beta$ & $\Delta t_{o}$ & & $P_{r}$ & $P_{r}$ & $T$ & $P_{r}$ & $T_{l}$ & $\beta$ & $\Delta t_{o}$ & & $P_{r}$ & $P_{r}$ & $T$ & $P_{r}$ & $T_{l}$ \\
\hline 0.002 & 0 & $S$ & 470 & 200 & 94.6 & 12 & 551 & 0.002 & 0 & $L$ & 632 & 208 & 93.6 & 14 & 370 \\
0.002 & 0.25 & $S$ & 408 & 156 & 84.3 & 13 & 20 & 0.002 & 0.5 & $S$ & 437 & 190 & 87.2 & 13 & 240 \\
0.004 & 0 & $S$ & 370 & 148 & 62.9 & 8.9 & 213 & 0.004 & 0 & $L$ & 303 & 139 & 66.0 & 9.0 & 164 \\
0.004 & 0.25 & $S$ & 430 & 160 & 55.0 & 9.3 & 109 & 0.004 & 0.5 & $S$ & 235 & 140 & 56.3 & 8.1 & 108 \\
0.01 & 0 & $S$ & 191 & 105 & 25.1 & 5.4 & 67 & 0.01 & 0 & $L$ & 386 & 163 & 28.5 & 6.4 & 80 \\
0.01 & 0.25 & $S$ & 238 & 86 & 24.2 & 4.2 & 59 & 0.01 & 0.5 & $S$ & 123 & 56 & 22.6 & 3.8 & 49 \\
0.05 & 0 & $S$ & 120 & 59 & 9.3 & 5.7 & 1070 & 0.05 & 0 & $L$ & 142 & 67 & 7.8 & 2.9 & 86 \\
0.05 & 0.25 & $S$ & 37 & 20 & 4.6 & 1.6 & 5 & 0.05 & 0.5 & $S$ & 96 & 37 & 6.4 & 2.3 & 21 \\
0.1 & 0 & $L$ & 23 & 9.1 & 6.1 & 0.6 & 229 & 0.1 & 0.25 & $S$ & 22 & 8.6 & 2.8 & 0.6 & 3 \\
0.1 & 0.5 & $S$ & 13 & 6.6 & 2.7 & 0.47 & 3 & 0.2 & 0 & $L$ & 7.4 & 3.5 & 3.6 & 0.27 & 119 \\
0.2 & 0.25 & $S$ & 20 & 4.5 & 1.9 & 0.39 & 2 & 0.2 & 0.5 & $S$ & 12 & 3.2 & 1.6 & 0.22 & 2 \\
0.4 & 0.25 & $S$ & 24 & 4.3 & 1.3 & 0.32 & 2 & 0.4 & 0.5 & $S$ & 13 & 3.5 & 1.4 & 0.22 & 2 \\
\hline
\end{tabular}

The results obtained are presented in Table 3. Again, $T$ is the mean time during which the perihelion distance $q$ was less than the semi-major axis of a given planet. In contrast to the asteroidal dust particles, the values of $T$ did not differ much between Venus, Earth, and Mars for the cometary dust particles. Below, $P_{r}=10^{6} P$ and $T_{l}$ is the longest lifetime of a particle in series of runs. For some runs at $\beta \geq 0.2$, all particles starting close to perihelion got hyperbolic orbits just after starting from the comet. These runs are not included in the Table.

All particles with $\beta \leq 0.01$ for $\Delta t_{o}=0$ or with $\beta \leq 0.2$ for $\Delta t_{o} \geq 0.25$ collided with the Sun. Relatively large values of $T_{l}$ for 'S' runs at $\beta=0.05$ and $\Delta t_{o}=0$ and for ' $\mathrm{L}$ ' runs at $\beta=0.1$ and $\Delta t_{o}=0$ were due to the particles that reached 2000 AU from the Sun. The values of $P_{r}$, $T$, and $T_{l}$ are greater for larger particles (i.e., for smaller $\beta$ ). The values of $P_{r}$ are greater for Venus than for Earth by a factor of 2 or more. Collision probabilities with Earth were greater by a factor of 10-20 than those with Mars and greater for particles starting at perihelion than aphelion. For the same values of $\beta$, the probability of cometary dust particles colliding with a terrestrial planet was several times smaller than for asteroidal dust particles, mainly due to the greater eccentricities and inclinations of the cometary particles. This difference is greater for larger particles.

The ratio of total times spent by cometary particles in inner-Earth, Aten, and Apollo orbits was about $1.5: 1: 2$, but varied from run to run.

\section{CONCLUSIONS}

We investigated collision probabilities of migrating asteroidal, cometary, and kuiperoidal dust particles with the terrestrial planets during the lifetimes of these particles. These probabilities were considerably greater for larger asteroidal and cometary particles, which is in accordance with the analysis of microcraters. The probability of collisions of cometary particles with the Earth is smaller than for asteroidal particles, and this difference is greater for larger particles. Almost all asteroidal particles with diameter $d \geq 4 \mu \mathrm{m}$ collided with the Sun. In almost all cases, inclinations $i<50^{\circ}$, and at $a>10 \mathrm{AU}$ the maximum $i$ was less for larger asteroidal particles. The spatial density of asteroidal particles decreases considerably at $a>3 \mathrm{AU}$, so the fraction of asteroidal particles among other particles beyond Jupiter's orbit is small. The peaks in the 
distribution of migrating asteroidal dust particles with semi-major axis corresponding to the $\mathrm{n}:(\mathrm{n}+1)$ resonances with Earth and Venus and the gaps associated with the 1:1 resonances with these planets are more pronounced for larger particles.

\section{ACKNOWLEDGEMENTS}

This work was supported by NRC (0158730), NASA (NAG5-10776), INTAS (00-240), and RFBR (01-02-17540).

\section{REFERENCES}

1. Liou, J.-C., H.A. Zook, \& S.F. Dermott. 1996. Kuiper belt dust grains as a source of interplanetary dust particles. Icarus. 124: 429-440.

2. Liou, J.-C. \& H. A. Zook. 1999. Signatures of the giant planets imprinted on the EdgeworthKuiper belt dust disk. Astron. J. 118: 580-590.

3. Gorkavyi, N.N., L.M. Ozernoy, T. Taidakova, \& J.C. Mather. 2000. Distribution of dust from Kuiper belt objects. http://arXiv.org/format/astro-ph/0006435.

4. Ozernoy, L.M. 2001. Physical modelling of the zodiacal dust cloud. In IAU Colloq. 204: The extragalactic infrared background and its cosmological implications. M. Harwit \& M.G. Hauser, Eds.: http://arXiv.org/format/astro-ph/0012033.

5. Moro-Martin, A. \& R. Malhotra. 2002. A study of the dynamics of dust from the Kuiper belt: spatial distribution and spectral energy distribution. Astron. J. 124: 2305-2321.

6. Liou, J.-C., H.A. Zook, \& A.A. Jackson. 1999. Orbital evolution of retrograde interplanetary dust particles and their distribution in the Solar system. Icarus. 141: 13-28.

7. Liou, J.-C., S.F. Dermott, \& Y.L. Xu. 1995. The contribution of cometary dust to the zodiacal cloud. Planet. Space Sci. 43: 717-722.

8. Reach, W.T., B.A. Franz, \& J.L. Weiland. 1997. The three-dimensional structure of the zodiacal dust bands. Icarus. 127: 461-484.

9. Kortenkamp, S.J. \& S.F. Dermott. 1998. Accretion of interplanetary dust particles by the Earth, Icarus. 135: 469-495.

10. Grogan, K., S.F. Dermott, \& D.D. Durda. 2001. The size-frequency distribution of the zodiacal cloud: Evidence from the Solar System dust bands. Icarus. 152: 251-267.

11. Fixsen, D. \& E. Dwek. 2002. The zodiacal emission spectrum as determined by COBE and its implications. Astrophys. J. 578: 1009-1014.

12. Hauser, M.G., F.C. Gillett, F.J. Low, et al. 1984. IRAS observations of the diffuse infrared background. Astrophys. J. 278: L15-L18.

13. Kelsall, T., J.L. Weiland, B.A. Franz, et al. 1998. The COBE diffuse infrared background experiment search for the cosmic infrared background. II. Model of the interplanetary dust cloud. Astrophys. J. 508: 44-73.

14. Grün, E., H. Kruger, M. Landgraf. 2000. Minor bodies in the outer solar system. In Proceedings of the ESO workshop (November 2-5, 1998, Garching, Germany). Springer. 99108.

15. Grün, E., H.A. Zook, H. Fechtig, \& R.H. Giese. 1985. Collisional balance of the meteoritic complex. Icarus. 62: 244-272.

16. Humes, D.H. 1980. Results of Pioneer 10 and 11 meteoroid experiments: Interplanetary and near-Saturn. J. Geophys. Res. 85: 5841-5852.

17. Grün, E. 1994. Dust measurements in the outer solar system. In IAU Symp. 160: Asteroids, Comets, Meteors 1993. A. Milani, M. Di Martino, \& A. Cellino, Eds.: 367-380. Kluwer Publishing. Dordrecht, Holland.

18. Burns, J.A., P.L. Lamy, \& S. Soter. 1979. Radiation forces on small particles in the Solar 
System. Icarus. 40: 1-48.

19. Ipatov, S.I. \& J.C. Mather. 2003. Migration of Jupiter-family comets and resonant asteroids to near-Earth space. This volume.

20. Ipatov, S.I., J.C. Mather, \& P. Taylor. 2003. Migration of asteroidal dust. In Abstracts of the 34th Lunar and Planetary Science Conference (March 17-21, 2003, League City, TX, USA). http://www.lpi.usra.edu/meetings/lpsc2003/pdf/1501.pdf.

21. Dermott, S.F., D.D. Durda, B.A.S. Gustafson, et al. 1994. Zodiacal dust bands. In IAU Symp. 160: Asteroids, Comets, Meteors 1993. A. Milani, M. Di Martino, \& A. Cellino, Eds.: 127-142. Kluwer Publishing. Dordrecht, Holland.

22. Dermott, S.F., S. Jayaraman, Y.L. Xu, et al. 1994. A circumsolar ring of asteroidal dust in resonant lock with the Earth. Nature. 369: 719-723.

23. Reach, W.T. 1992. Zodiacal emission. III - Dust near the asteroid belt. Astrophys. J. 392: 289-299.

24. Ipatov, S.I., J.C. Mather, and P.A. Taylor. 2003. Migration of asteroidal dust particles, In Proc. of the international conference "New trends in astrodynamics and applications" (20-22 January 2003, University of Maryland, College Park). CD-ROM (http://arXiv.org/format/astro-ph/0303398). 